\title{
Overview of the optomechanical design of the LUVOIR instruments
}

\author{
James Corsetti ${ }^{\mathrm{a}}$, Matthew Bolcar ${ }^{\mathrm{a}}$, Julie Crooke ${ }^{\mathrm{a}}$, Brian Fleming ${ }^{\mathrm{b}}$, Kevin France ${ }^{\mathrm{b}}$, Joseph \\ Generie $^{\mathrm{a}}$, Qian Gong ${ }^{\mathrm{a}}$, Tyler Groff ${ }^{\mathrm{a}}$, Jason Hylan ${ }^{\mathrm{a}}$, Andrew Jones ${ }^{\mathrm{a}}$, Lia Sacks ${ }^{\mathrm{a}}$, Garrett \\ West $^{\mathrm{a}}$, Kan Yang ${ }^{\mathrm{a}}$, Neil Zimmerman ${ }^{\mathrm{a}}$ \\ ${ }^{a}$ NASA GSFC, 8800 Greenbelt Rd, Greenbelt, MD 20771; \\ ${ }^{b}$ University of Colorado Boulder, Department of Astrophysical and Planetary Sciences, 2000 \\ Colorado Ave, Boulder, CO 80309;
}

\begin{abstract}
The Large Ultraviolet/Optical/Infrared Surveyor (LUVOIR) is a space telescope being submitted for review to the 2020 Decadal Survey in Astronomy and Astrophysics. Its science objectives include both direct imaging and spectral characterization of habitable exoplanets around sun-like stars, the study of planet, star, and galaxy formation, the transfer of matter between different galaxies, and the remote sensing of objects within the Solar System. Two architectures have been designed: a $15 \mathrm{~m}$ diameter on-axis telescope (LUVOIR-A) and an $8 \mathrm{~m}$ off-axis telescope (LUVOIR-B).

This paper discusses the opto-mechanical design of the three LUVOIR instruments: the High Definition Imager (HDI), the LUVOIR UV Multi-object Spectrograph (LUMOS), and the Extreme Coronagraph for Living Planetary Systems (ECLIPS). For both the LUVOIR-A and LUVOIR-B variants of each instrument, optical design specifications are presented including first-order constraints, packaging requirements, and optical performance metrics. These factors are used to illustrate the final design of each instrument and LUVOIR as a whole. In addition to the optical designs, mechanical models are presented for each instrument showing the optical mounts, mechanisms, support structure, etc.
\end{abstract}

Keywords: Times Roman, image area, acronyms, references

\section{INTRODUCTION}

We note that portions of this manuscript appear in the LUVOIR Study Final Report, which is publicly available at https://luvoirtelescope.org. ${ }^{[1]}$

The Large Ultraviolet/Optical/Infrared Surveyor (LUVOIR) is a large-scale space telescope and one of four concepts being submitted for the 2020 Decadal Survey in Astronomy and Astrophysics. ${ }^{[2]}$ Two architectures have been studied, the 15m-diameter on-axis LUVOIR-A as shown in Figure 1 and the 8m-diameter off-axis LUVOIR-B as shown in Figure $2 .{ }^{[3]}$ Both telescopes are segmented and three-mirror anastigmats with the same $10 \times 8$ arcminute field of view.
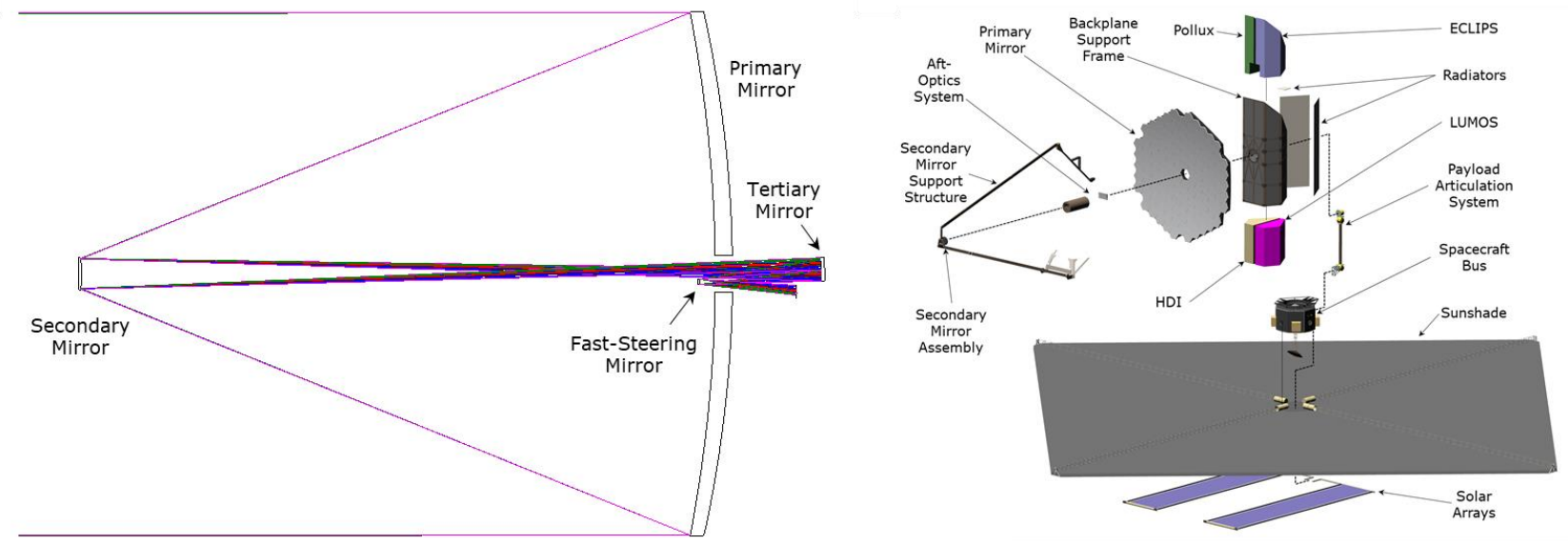

Figure 1. Left: CODEV ${ }^{\circledR}$ optical model of LUVOIR-A telescope. Right: exploded view of observatory to highlight instrument locations relative to one another and telescope. 

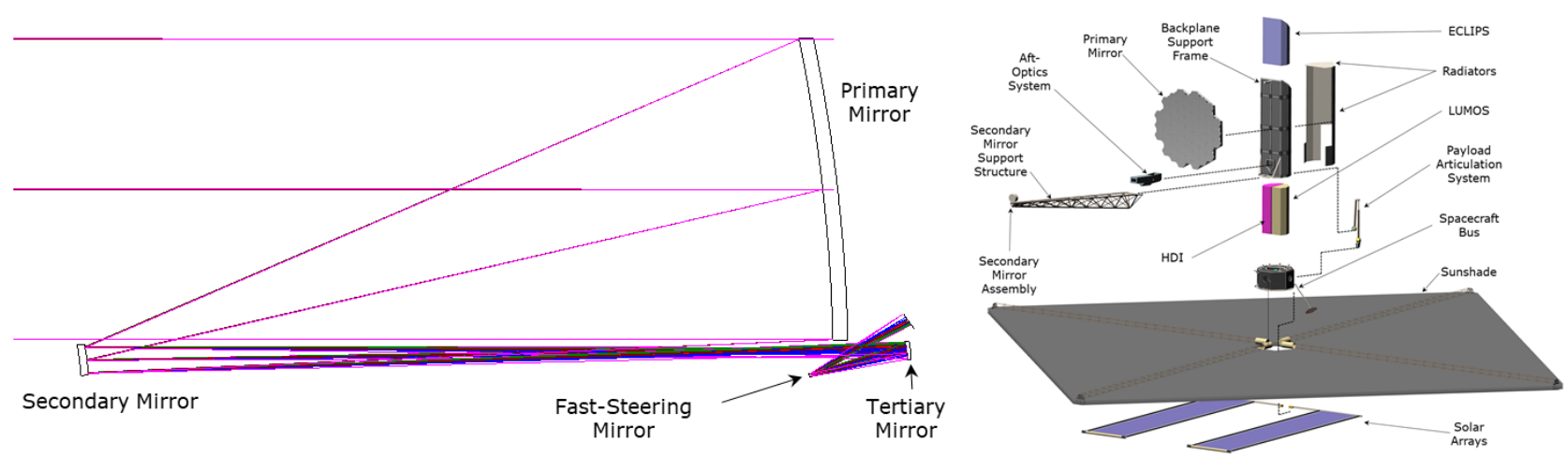

Figure 2: Left: $\mathrm{CODEV}^{\circledR}$ optical model of LUVOIR-B telescope. Right: exploded view of observatory to highlight instrument locations relative to one another and telescope.

\section{HIGH DEFINITION IMAGER (HDI)}

\subsection{Overview}

LUVOIR's High Definition Imager (HDI) is the observatory's primary instrument for imaging over a wide field of view. HDI operates over a large waveband between the ultraviolet (UV) and near-infrared (NIR), being split between two channels: the UV-visible (UVIS) from 200 to $\sim 1000 \mathrm{~nm}$ and the NIR between $\sim 800$ and $2500 \mathrm{~nm}$. This section discusses the layout and optical performance of the versions of the instrument compatible with LUVOIR-A (HDI-A) and LUVOIR-B (HDI-B).

\subsection{Requirements and Assumptions}

The optical and detector specifications for HDI-A and HDI-B are summarized in Table 1. Calculations for both the Nyquist-sampled plate scale and diffraction-limited spot size (DLSS) are carried out using a wavelength of $0.5 \mu \mathrm{m}$ and $1 \mu \mathrm{m}$ for the UVIS and NIR channels, respectively. In each case, the effective focal length of HDI is calculated to give a Q-sampling value of 4.88 (each diffraction limited spot subtends 4.88 pixels). It is desired to have an exit pupil diameter of approximately $50 \mathrm{~mm}$ after the pupil relay mirrors to limit the required size of the optical elements within the filter wheels.

Table 1. Optical and detector specifications for HDI-A and HDI-B

\begin{tabular}{|c|c|c|c|c|c|}
\hline \multirow{2}{*}{ Parameter } & \multirow{2}{*}{ Units } & \multicolumn{2}{|c|}{ HDI-A } & \multicolumn{2}{c|}{ HDI-B } \\
\cline { 3 - 6 } & & UVIS & NIR & UVIS & NIR \\
\hline Bandpass & $\mu \mathrm{m}$ & $0.2-1.0$ & $0.8-2.5$ & $0.2-1.0$ & $0.8-2.5$ \\
\hline Aperture Diameter (D) & $\mathrm{m}$ & 15 & 15 & 8 & 8 \\
\hline$f$-number & -- & 26 & 20 & 26 & 20 \\
\hline Focal Length & $\mathrm{m}$ & 390 & 300 & 208 & 160 \\
\hline Field of View & arcmin & $2.91 \times 2.11$ & $2.94 \times 2.17$ & $2.69 \times 1.78$ & $2.71 \times 1.79$ \\
\hline Platescale $(\lambda / 2 \mathrm{D})$ & mas/pixel & 3.44 & 6.88 & 6.45 & 12.89 \\
\hline DLSS & $\mu \mathrm{m}$ & 31.72 & 48.80 & 31.72 & 48.80 \\
\hline RMS Pointing Stability & $1 \sigma \mathrm{mas}$ & 0.43 & 0.86 & 0.81 & 1.61 \\
\hline RMS Wavefront Error & $\mathrm{nm}$ & $<35$ & $<71$ & $<35$ & $<71$ \\
\hline Detector Type & -- & $\mathrm{CMOS}$ & $\mathrm{HgCdTe}$ & $\mathrm{CMOS}$ & $\mathrm{HgCdTe}$ \\
\hline Pixel Width & $\mu \mathrm{m}$ & 6.5 & 10 & 6.5 & 10 \\
\hline Q-sampling & -- & 4.88 & 4.88 & 4.88 & 4.88 \\
\hline Detector Width & pixels & 8192 & 4096 & 8192 & 4096 \\
\hline Array Tiling & -- & $6 \times 4$ & $6 \times 4$ & $3 \times 2$ & $3 \times 2$ \\
\hline Total Number of Pixels & Gigapixels & 1.611 & 0.403 & 0.403 & 0.101 \\
\hline
\end{tabular}




\subsection{System Description}

Figure 3 and Figure 4 show the optical block diagram and layout respectively of HDI-A. As shown, a fold mirror is located after the focal surface of the OTE to direct the light to a pair of pupil relay mirrors. From there, the light passes through a channel selection mechanism which determines which channel(s) is/are operating at any given time. The channel selection mechanism has a total of five optical elements and therefore five modes:

NIR Transmissive: sends all light from the optical telescope to the NIR channel. This position also allows light from an internal calibration source to be directed into the UVIS channel.

UVIS Reflective: sends all light from the optical telescope to the UVIS channel. This position also allows light from an internal calibration source to be directed into the NIR channel.

50/50 Beamsplitter: allows for simultaneous operation of both channels over the entire instrument bandpass, but at a reduced throughput. It is assumed that such a coating will have about a $2 \%$ efficiency loss (so that each of the two respective bands transmits or reflects approximately $98 \%$ of the incident light, resulting in 49/49 splitting in reality).

Dichroic Beamsplitter: allows for simultaneous operation of the UVIS channel between $400 \mathrm{~nm}$ and $800 \mathrm{~nm}$, and the NIR channel between $800 \mathrm{~nm}$ and $1.6 \mu \mathrm{m}$. Similar to the 50/50 beamsplitter, it is assumed that the dichroic beamsplitter coating has about a $2 \%$ loss.

Optimized UV Reflective: sends all of the light from the optical telescope into the UVIS channel, but emphasizes UV throughput over broadband operation. For this coating, the intention is to provide maximum reflectance in the band between 200 and $400 \mathrm{~nm}$.

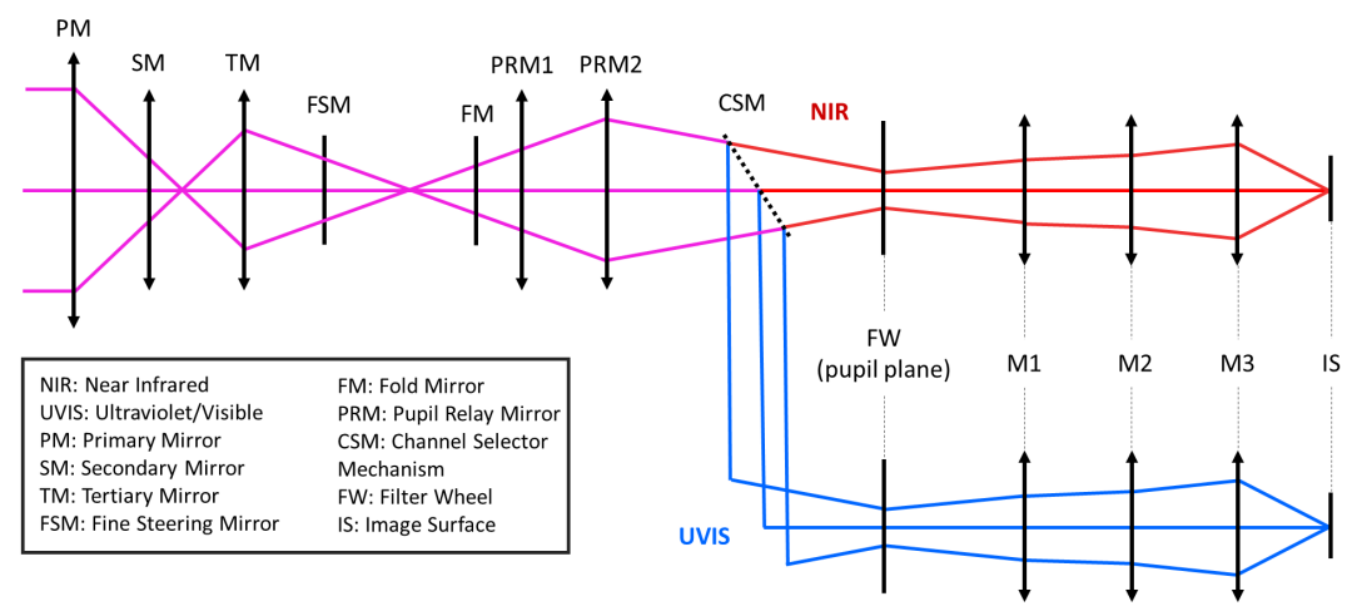

Figure 3. Optical block diagram for HDI-A. The block diagram is identical for HDI-B save the presence of the fold mirror after the FSM. 


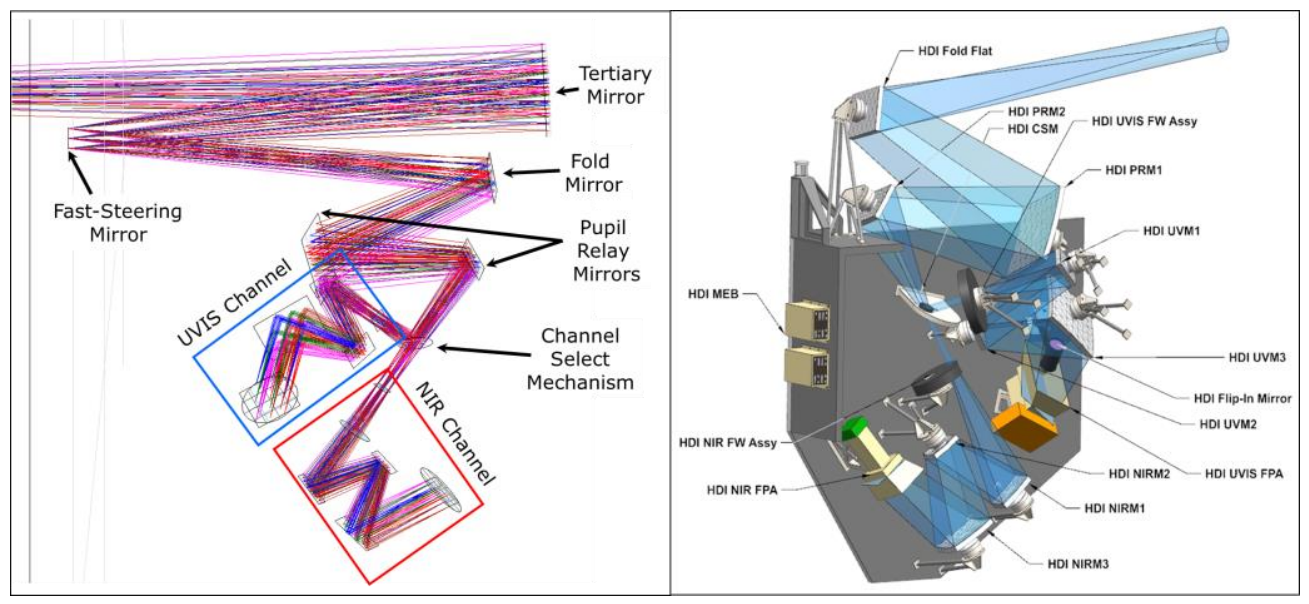

Figure 4. HDI-A design. Optical ray trace on left and mechanical model on right.

After the light reflects off of the two pupil relay mirrors and is spectrally split by the channel selection mechanism, the aforementioned pupils are formed farther downstream in the optical trace. An optical filter wheel is placed in each channel at the location of the pupil as shown in Figure 4. The UVIS channel accommodates 39 spectral filters, two grisms, and an assortment of weak lenses and dispersed Hartmann sensors to support image-based wavefront sensing operations. The NIR channel accommodates 24 spectral filters and two grisms. It is useful to place the filters at a pupil since the footprint of the beam is minimized there, reducing the required size of the filters. Note that in the current CODEV $®$ optical model, the channel selection mechanism and the spectral filters are modeled are simple surfaces having no physical thickness nor specific material/refractive index properties. In further design iterations actual optical windows will be modeled rather than surfaces. After the filter wheel in each channel, the light enters a three-mirror system that images the observed field of view to the image surface/detector plane.

HDI-B is very similar in concept and layout to HDI-A. The only difference between the two designs (other than variations in the surface prescriptions of the individual optical elements) is that HDI-B lacks the flat fold mirror that HDI-A has to pickoff the light reflected from the FSM. Instead, the first of HDI-B's two pupil relay mirrors serves this purpose (in addition to helping form the pupils for the filter wheels just as in HDI-A). This reduction in degrees of freedom for the optical designer does make the design of HDI-B more challenging than HDI-A from an aberration correction point of view but at the same time decreases the required size of the instrument. Figure 5 shows the layout for HDI-B.

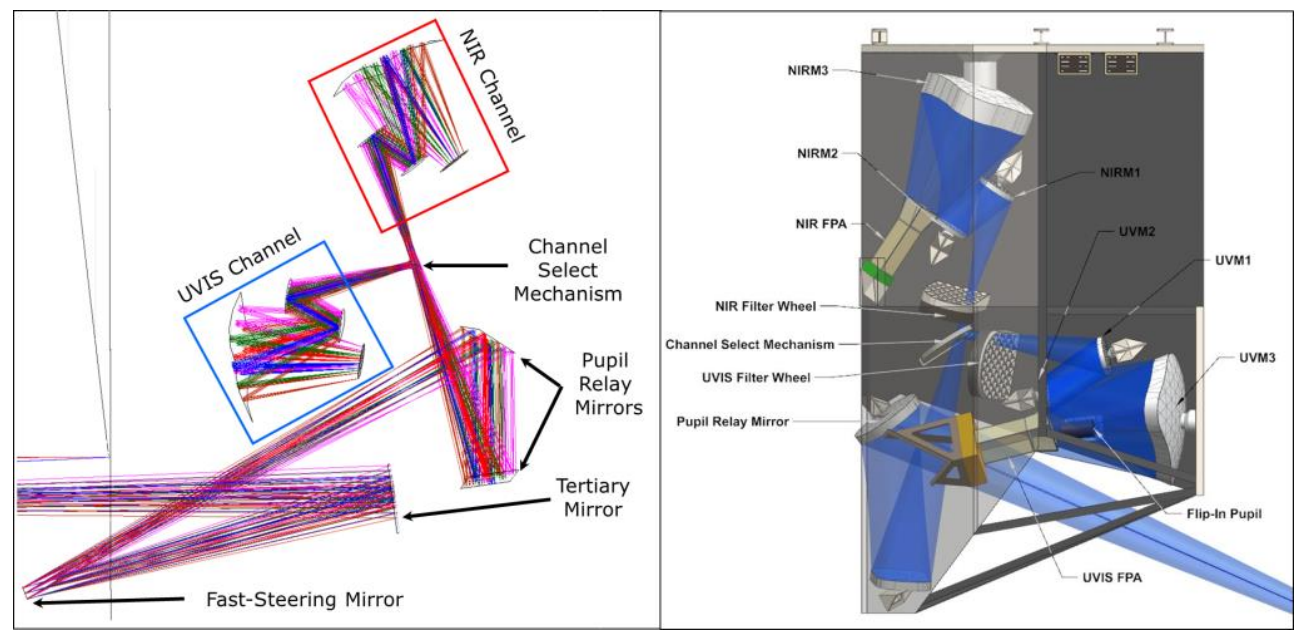

Figure 5. HDI-B design. Optical ray trace on left and mechanical model on right. 
In order to achieve improved imaging performance, a number of HDI's reflective elements are designed using 'freeform' optical surfaces. Such surfaces can be described by a polynomial that varies in both the $\mathrm{x}$ and $\mathrm{y}$ directions. Mathematically, the surface sag of such a mirror is described by

$$
z(s)=\frac{c s^{2}}{1+\sqrt{1-(1+k) c^{2} s^{2}}}+\sum_{j=2}^{66} C_{j} x^{m} y^{n} \quad \text { and } \quad j=\frac{(m+n)^{2}+m+3 n}{2}+1
$$

where $\mathrm{z}$ is the surface sag as a function of the radial coordinate $s, c$ is the curvature (inverse of the radius of curvature, $r$ ) of the surface, $k$ is the conic coefficient, and $C j$ are the series of coefficients composing the aforementioned xy polynomial. These freeform surfaces improve aberration correction and therefore imaging performance over a larger field of view as compared to conventional rotationally symmetric optics. In both HDI-A and HDI-B the two pupil relay mirrors, the three mirrors that compose the NIR imaging channel, and the three mirrors that compose the UVIS imaging channel are all freeform optical surfaces. In HDI-A, the tertiary mirror is one as well. The maximum such sag departure for a freeform mirror in HDI-A is 3.98mm on NIR Mirror \#3 while the maximum such sag departure for a freeform mirror in HDI-B is $6.79 \mathrm{~mm}$ on UVIS Mirror \#3. Further design iterations of HDI-A and HDI-B can be carried out in the future with constraints in place to limit this maximum value of sag departure from a best fit sphere across the surface of the optic. Overall, the HDI-B mirrors have larger surface sags across the mirror apertures than do the HDI-A mirrors. This is likely in part due to the fact that HDI-B does not have the flat fold mirror that HDI-A does have (as mentioned above) and also has one less freeform surface than HDI-A (the tertiary) for correcting aberrations.

\subsection{Summary of Performance}

The primary optical performance requirement for HDI is listed in Table 1 with RMS wavefront error specified to be less than 35 and $71 \mathrm{~nm}$ for the UVIS and NIR channels, respectively. Figure 6 shows the RMS wavefront error plotted as a function of field of view for both channels of the HDI-A design. While the performance requirement is met across the majority of the field of view for the UVIS channel, there are regions (mainly in the lower left corner) that are above the $35 \mathrm{~nm}$ threshold. This region is worse than the others in terms of optical performance because it is farther off-axis (optical aberrations generally come into play more with farther off-axis field points). The performance can be improved on with more design iterations that further optimize the prescription of the OTE mirrors and/or the instrument's field of view allocation. Both of these steps would improve the wavefront first entering HDI, making aberration-correction comparatively easier. The NIR channel meets the wavefront performance requirement across the entire field of view. It should also be noted that the $3 \times 2$ arcminute field of view that performance is evaluated over is larger than the requirements included in Table 1.

Figure 7 shows the same analysis as Figure 6 but for HDI-B. Please note the change in scale between the two sets of plots for HDI-A and HDI-B. Like for HDI-A, while the UVIS channel does meet wavefront performance across the majority of the field of view, there are regions where the value is greater than $35 \mathrm{~nm}$. Similarly, the NIR channel does meet the performance specification across the majority of the full field of view but there are spots (mainly towards the edge of the FOV) where it does not. Overall, the wavefront performance for HDI-B is comparatively worse than that of HDI-A. This is accounted for, at least in part, due to HDI-B not having the separate flat pickoff mirror that HDI-A does, requiring the first pupil relay mirror to address more design constraints itself. With more time and further optimization, the design presented in this document could certainly be improved for imaging performance. Additionally, a more indepth design study could be carried out with the specific purpose of reducing the design complexity by decreasing the number of xy polynomial freeform surfaces in favor of simpler off-axis conical surfaces that are easier to manufacture and test. The average, maximum, and minimum values of the RMS wavefront error for both HDI-A and HDI-B are tabulated in Table 2 in units of nanometers.

Table 2. Summary of RMS wavefront error performance for HDI-A and HDI-B. All values are in nm.

\begin{tabular}{|c|c|c|c|c|}
\cline { 2 - 5 } \multicolumn{1}{c|}{} & \multicolumn{2}{c|}{ HDI-A } & \multicolumn{2}{c|}{ HDI-B } \\
\cline { 2 - 5 } \multicolumn{1}{c|}{} & UVIS & NIR & UVIS & NIR \\
\hline Requirement & $<35$ & $<71$ & $<35$ & $<71$ \\
\hline Average & 25.8 & 25.7 & 28.3 & 29.3 \\
\hline Maximum & 53.5 & 59.0 & 104.6 & 104.9 \\
\hline Minimum & 9.5 & 9.9 & 6.6 & 9.4 \\
\hline
\end{tabular}



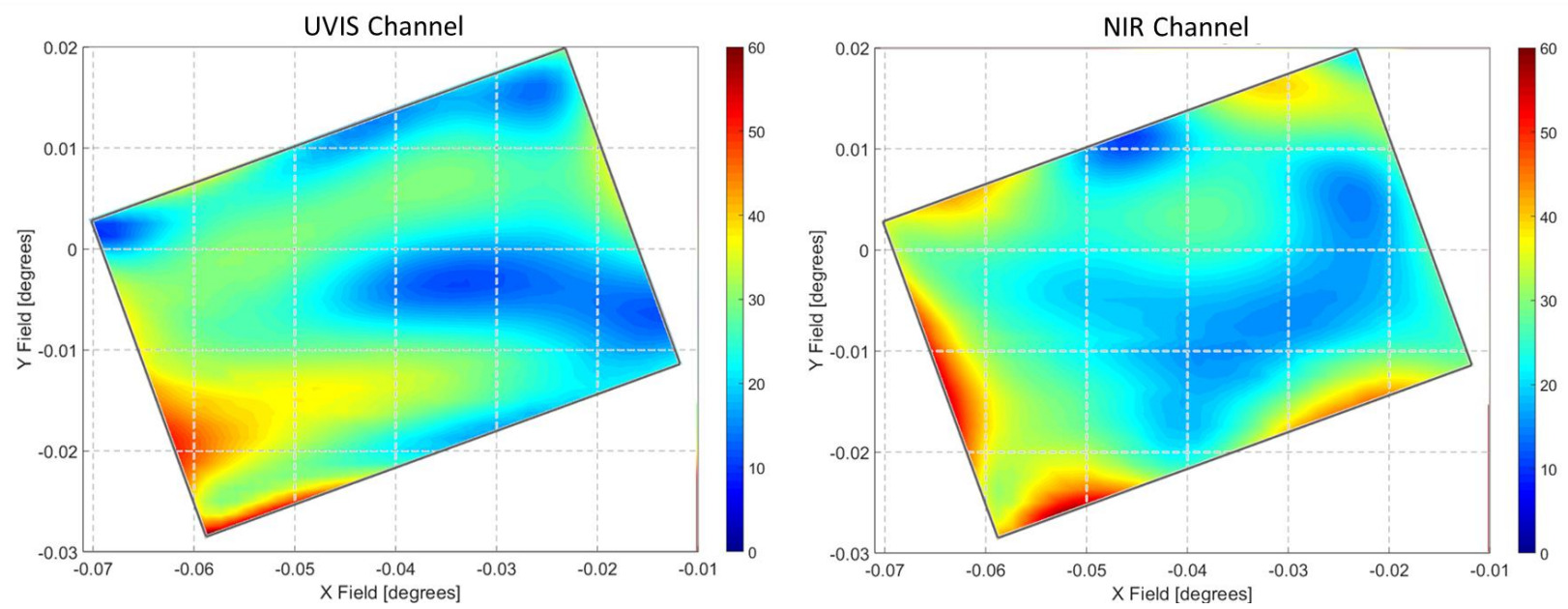

Figure 6. RMS Wavefront Error (measured in nm) for HDI-A.
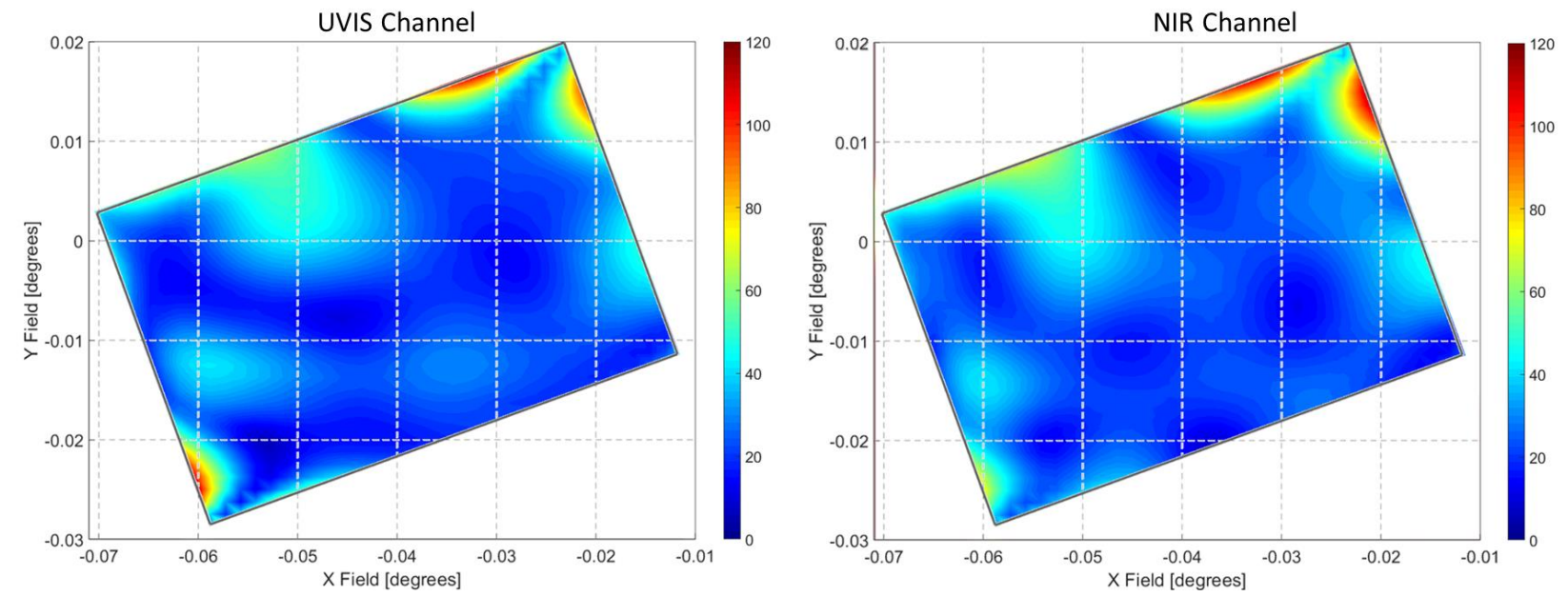

Figure 7. RMS Wavefront Error (measured in nm) for HDI-B.

\section{LUVOIR ULTRAVIOLET MULTI-OBJECT SPECTROGRAPH (LUMOS)}

\subsection{Narrative Overview}

The LUVOIR Ultraviolet Multi-Object Spectrograph (LUMOS) is an instrument capable of both point source and multiobject spectroscopy (MOS) as well as imaging in the ultraviolet spectrum. The instrument will provide low ( $\mathrm{R}=8,000$ 18,000) and medium $(\mathrm{R}=30,000-60,000)$ resolution modes across the far-ultraviolet (FUV: $100-200 \mathrm{~nm})$, nearultraviolet (NUV: $200-400 \mathrm{~nm}$ ), and visible (VIS: $400-1000 \mathrm{~nm}$ ) windows, and a very low resolution mode (R = 500) for spectroscopic investigations of extremely faint objects in the FUV. LUMOS also allows for imaging in the FUV. This section discusses the layout and optical performance of the versions of the instrument compatible with LUVOIR-A (LUMOS-A) and LUVOIR-B (LUMOS-B).

\subsection{Requirements and Assumptions}

The optical requirements and performance results for LUMOS are shown in

Table 3. Further details on the science cases for the instrument have been previously discussed. ${ }^{[4]}$ 
Table 3: Required and achieved performance and first order specifications for both LUMOS-A and LUMOS-B. Results for the FUV, NUV and VIS MOSs and FUV imager are described.

\begin{tabular}{|c|c|c|c|c|c|c|c|c|c|}
\hline Instrument Parameter & G120M & G150M & G180M & G155L & G145LL & G165LL & G300M & G700L & FUV Imager \\
\hline Optimized Spectral Bandpass ( $\mathrm{nm}$ ) & $100-140$ & $130-170$ & $160-200$ & $100-200$ & $100-200$ & $110-200$ & $200-400$ & $400-1000$ & $100-200$ \\
\hline Actual Spectral Bandpass $(\mathrm{nm})$ & $93-159$ & $111-189$ & $141-219$ & $93-267$ & $93-210$ & $110-270$ & $193-460$ & $340-1000$ & $100-200$ \\
\hline Field of View (FOV) & $2^{\prime} \times 2^{\prime}$ & $2^{\prime} \times 2^{\prime}$ & $2^{\prime} \times 2^{\prime}$ & $2^{\prime} \times 2^{\prime}$ & $2^{\prime} \times 2^{\prime}$ & $2^{\prime} \times 2^{\prime}$ & $\begin{array}{c}1.5^{\prime} \times 2^{\prime}(\mathrm{A}) \\
2^{\prime} \times 2^{\prime}(\mathrm{B})\end{array}$ & $\begin{array}{c}1.5^{\prime} \times 2^{\prime}(\mathrm{A}) \\
2^{\prime} \times 2^{\prime}(\mathrm{B})\end{array}$ & $\begin{array}{c}1.2^{\prime} \times 2^{\prime}(A) \\
2^{\prime} \times 2^{\prime}(B)\end{array}$ \\
\hline Spectral Resolving Power (Objective: $\lambda / \Delta \lambda$ ) & 30,000 & 30,000 & 30,000 & 8,000 & 500 & 500 & 20,000 & 15,000 & $-\ldots$ \\
\hline Imaging Resolution (Objective: mas) & 50 & 50 & 50 & 50 & 50 & 50 & 50 & 50 & 50 \\
\hline \multicolumn{10}{|c|}{ LUMOS A } \\
\hline Effective Area $\left(\right.$ Peak, $\mathrm{cm}^{2}$ ) & 311,540 & 136,780 & 160,610 & 304,300 & 184,880 & 311,545 & 324,950 & 302,750 & $\sim 100,000$ \\
\hline Effective Area (Central Wavelength, $\mathrm{cm}^{2}$ ) & 293,270 & 102,750 & 160,610 & 91,250 & 31,460 & 91,240 & 247,630 & 266,690 & $\sim 100,000$ \\
\hline Grating Ruling Density (groove/mm) & 1,950 & 2,050 & 2,020 & 815 & 21 & 43 & 450 & 150 & --- \\
\hline Average Resolving Power (All Microshutters) & 29,875 & 35,990 & 40,991 & 14,063 & 408 & 820 & 20,531 & 14,466 & -.-- \\
\hline Average Resolving Power (Best $\left.1^{\prime} \times 1^{\prime}\right)$ & 39,276 & 47,004 & 54,812 & 17,508 & 583 & 1,170 & 28,431 & 19,950 & $-\cdots$ \\
\hline Average Angular Resolution (All Microshutters) & 37 & 43 & 39 & 42 & 38 & 38 & 31 & 33 & 50 \\
\hline Average Angular Resolution (Best 1' x 1') & 28 & 32 & 30 & 34 & 26 & 26 & 19 & 21 & 42 \\
\hline \multicolumn{10}{|c|}{ LUMOS B } \\
\hline Effective Area (Peak) & 86,140 & 37,820 & 44,410 & 84,140 & 51,120 & --- & 89,850 & 83,710 & $\sim 27,500$ \\
\hline Effective Area (Central Wavelength) & 81,090 & 28,410 & 44,410 & 25,230 & 8,700 & 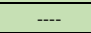 & 68,470 & 73,740 & $\sim 27,500$ \\
\hline Grating Ruling Density (groove/mm) & 2,555 & 2,630 & 2,610 & 1,060 & 50 & $-\cdots$ & 865 & 349 & --- \\
\hline Average Resolving Power (All Microshutters) & 29,422 & 36,863 & 49,699 & 13,699 & 455 & $-\cdots$ & 19,983 & 17,604 & $-\cdots$ \\
\hline Average Resolving Power (Best 1' x 1') & 40,264 & 52,223 & 59,310 & 17,414 & 537 & ---- & 32,745 & 28,223 & 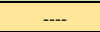 \\
\hline Average Angular Resolution (All Microshutters) & 41 & 42 & 43 & 48 & 28 & $-\cdots$ & 25 & 48 & 48 \\
\hline Average Angular Resolution (Best 1' x 1') & 31 & 32 & 33 & 39 & 23 & $-\cdots$ & 23 & 41 & 40 \\
\hline
\end{tabular}

\subsection{System Description}

Both LUMOS-A and LUMOS-B contain three channels: a FUV multi-object spectrograph (MOS), a NUV/VIS MOS, and an FUV imager. The layouts of each of the three channels for each of the two designs are further detailed in this section. Figure 8 shows the optical block diagram for LUMOS-A while Figure 9 shows the Zemax optical layout of LUMOS-A with all three channels displayed at once with the rays colored by configuration number in the software. To see the detail of each channel, Figure 9 shows the FUV MOS, NUV/VIS MOS, and FUV imaging channels separately. In Figure 9, the rays are colored by wavelength in order to better identify the wavelength-dependent sections of the beam path. After light reflects off of the fast steering mirror (FSM) in the optical telescope assembly (OTA) into LUMOS, it passes through a $2 \times 2$ grid of microshutter arrays (MSA) which defines the field of view for the MOS. Light passing through the MSA is folded into the spectrograph by a nominally fixed (with fine focus control for calibration) convex biconic optic: the MOS Fold Mirror (FM) 1. A second fixed aberration-correcting toroidal mirror (MOS FM2) directs the beam to the FUV or NUV grating wheel. MOS FM 2 is fixed, with the NUV grating wheel picking off the beam en route to the FUV grating wheel if those modes are selected.

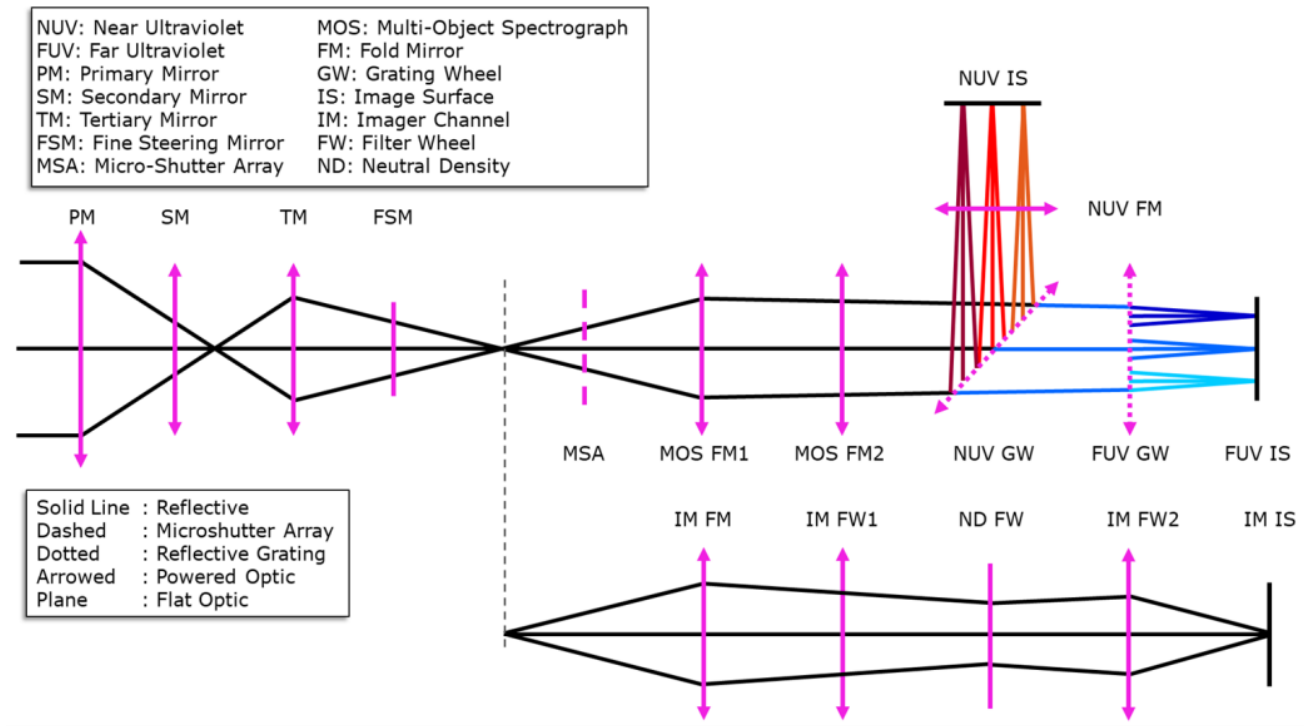

Figure 8. Optical block diagram for LUMOS-A. 


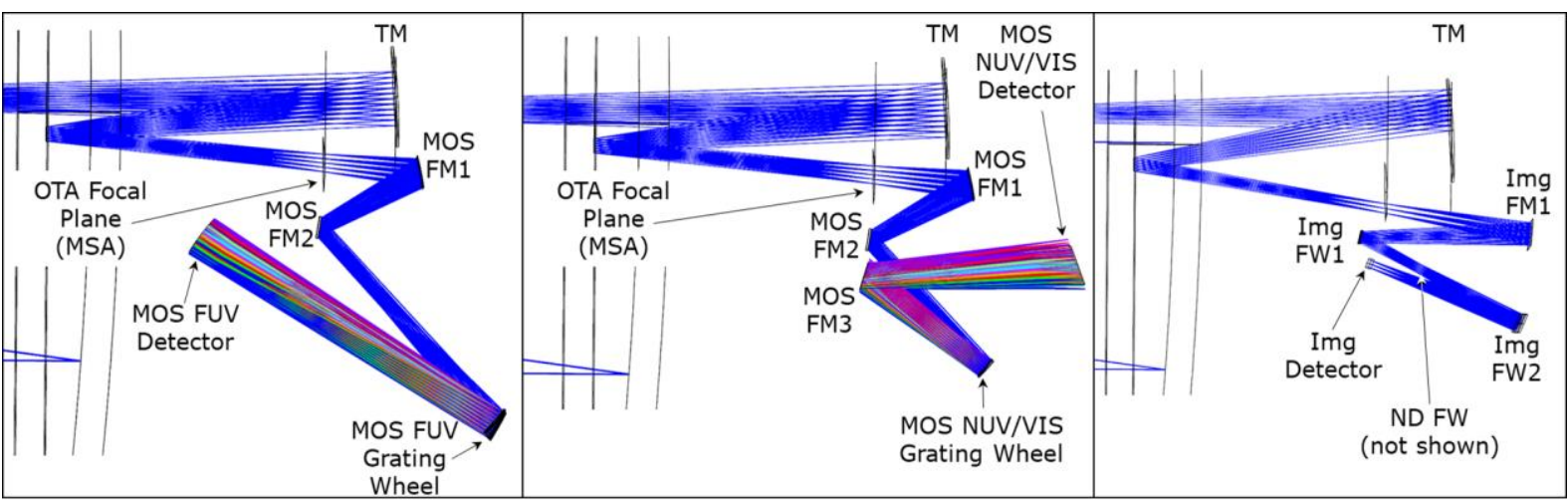

1. FUV multi-object spectrograph

2. NUV/VIS multi-object spectrograph

3. FUV imager

Figure 9. Layouts of the three individual channels for LUMOS-A. From left to right: far ultraviolet (FUV) multi-object spectrograph (MOS), near ultraviolet (NUV) MOS, and FUV imager.

\subsubsection{FUV and NUV multi-object spectroscopy}

The FUV MOS modes include medium, low, and very low spectral resolution settings contained within the FUV grating wheel $(\mathrm{GW})$ that all maintain sub-MSA shutter imaging performance, essentially creating an array of long-slits that can be used for point-source spectroscopy or extended source imaging spectroscopy. The gratings are set within a wheel mechanism and selected by wheel position. The medium resolution gratings provide spectral resolving power, $\mathrm{R}$, values between about 4,000 and 55,000 (depending on the grating setting and field location) over the best $1 \mathrm{x} 1$ ' field-of-view (total field of view is $2^{\prime} \times 2^{\prime}$ ). The grating names are chosen in analogy with the HST naming convention, where ' $G$ ' refers to a first order grating, the middle three numbers are roughly the central wavelength in nanometers, and the final letter ('M', 'L', or 'LL') denotes the spectral resolution of the mode (Medium, Low, or Low-Low). See

Table 3 for more details on requirements and performance.

All of the FUV MOS modes (except G145LL) are focused onto a 2 x 1 array of large-format microchannel plate detectors, with the full spectral bandpass spanning two detector faces. The shorter wavelengths of each mode fall onto a short-wavelength optimized MCP with a CsI photocathode (like HST-COS), while the longer wavelengths fall onto a $\mathrm{GaN}$ photocathode MCP optimized for longer wavelengths, but not sensitive to wavelengths shorter than $110 \mathrm{~nm}$. The G145LL focuses the entire 2' x 2' FOV onto the short wavelength optimized MCP, while the G165LL focuses the FOV onto the longer wavelength optimized MCP.

In order to access the NUV/VIS MOS modes, the NUV GW mechanism (located between MOS FM2 and the FUV GW, see Figure 10 which shows the CAD models for both versions of LUMOS) rotates the desired grating into place, preventing the light from reaching the FUV GW. After reflecting off of the NUV GW, the light is directed towards the NUV fold mirror (NUV FM) which in turn sends the light towards the channel's focal plane array. The NUV/VIS grating modes are G300M and G700L, the details of which are included in

Table 3 .

\subsubsection{FUV Imager}

The majority of the LUVOIR imaging science is addressed through the HDI instrument (200nm $-2.5 \mu \mathrm{m})$, and LUMOS provides a complimentary FUV imaging capability from $100-200 \mathrm{~nm}$. The LUMOS-A FUV imaging aperture subtends a 1.2' x 2' FOV, and is physically offset from the MOS MSA array. Light from the OTA enters this channel through an unobstructed open aperture with separate pickoff mirrors and subsequent beam paths from the FUV MOS. This is not the case for LUMOS-B which is discussed below. The incident light is folded into the imaging channel off of a biconic convex fold optic with fine piston tip/tilt focus control similar to MOS FM1 (Imager Fold Mirror, IM FM) and then through two identical reflective filter wheel assemblies (IM FW1 and IM FW2) that serve to define the imaging bandpass in this mode. A neutral-density filter wheel (ND FW, not included in the Zemax model) is inserted between the two filter wheels to accommodate FUV bright object protection. The images are then recorded on a single $170 \mathrm{~mm} \mathrm{x}$ $110 \mathrm{~mm}$ CsI photocathode MCP detector. Figure 10 shows the layout of LUMOS-A in further detail with special attention paid to detail the positions within the design that will require multiple elements and therefore a mechanism, namely the grating and filter wheels. 


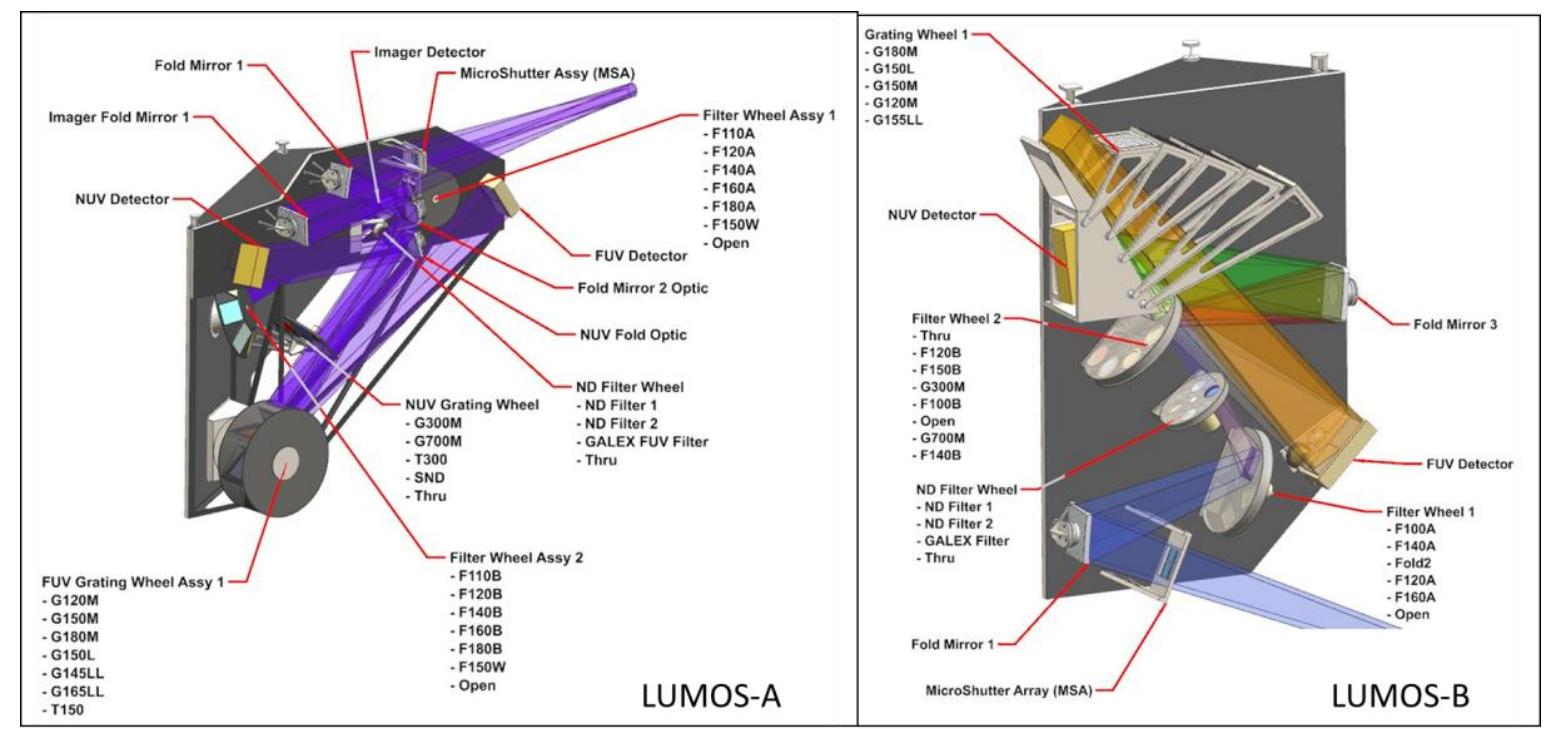

Figure 10. LUMOS-A and LUMOS-B grating and filter wheel mechanisms

\subsubsection{LUMOS -B}

It should be noted that LUMOS is the only of the three LUVOIR instruments to have significant differences in optical layout between the A and B configurations. This was the result of the limited volume available in LUMOS-B as compared to LUMOS-A. Figure 11 shows the optical block diagram for LUMOS-B. Figure 12 shows the FUV MOS, NUV MOS, and FUV imaging channels separately. As mentioned before, there are not separate pickoffs for the MOS and imaging channels in LUMOS-B. All the light entering LUMOS from the OTA does so by first passing through the MSA before being reflected by a fold mirror. While the second optic after the MSA in LUMOS-A was a single mirror (MOS FM2), Figure 10 and Figure 12 show that in LUMOS-B, it is a mechanism (filter wheel 1) that switches between a number of elements depending on the mode of operation. For MOS modes, a simple broadband reflective mirror rotates into place, while a narrowband filter is used for imaging. After reflecting off of an element in FW1 (and passing through the neutral density filter wheel), the light follows one of the following paths depending on the mode of operation:

FUV MOS: the light reflects off of the FUV grating wheel before reaching the FUV focal plane array.

NUV MOS: the light reflects off of an element within a mechanism that holds elements for both the NUV MOS and imaging modes (NUV GW/FW2). For NUV MOS, the light then reflects off of the NUV fold mirror before reaching the NUV FPA.

Imaging: the light reflects off an element within the NUV GW/FW2 mechanism. For imaging, the light is directed towards the FUV FPA which tilts into one of two positions depending on if the imaging or FUV MOS mode is being utilized. The short wavelength filter (F110) is imaged onto the short wavelength optimized MCP, while the other filters are imaged onto the long wavelength sensitive MCP. 


\begin{tabular}{|ll|}
\hline NUV: Near Ultraviolet & MOS: Multi-Object Spectrograph \\
FUV: Far Ultraviolet & FM: Fold Mirror \\
PM: Primary Mirror & GW: Grating Wheel \\
SM: Secondary Mirror & IS: Image Surface \\
TM: Tertiary Mirror & IM: Imager Channel \\
FSM: Fine Steering Mirror & FW: Filter Wheel \\
MSA: Micro-Shutter Array & ND: Neutral Density \\
\hline
\end{tabular}

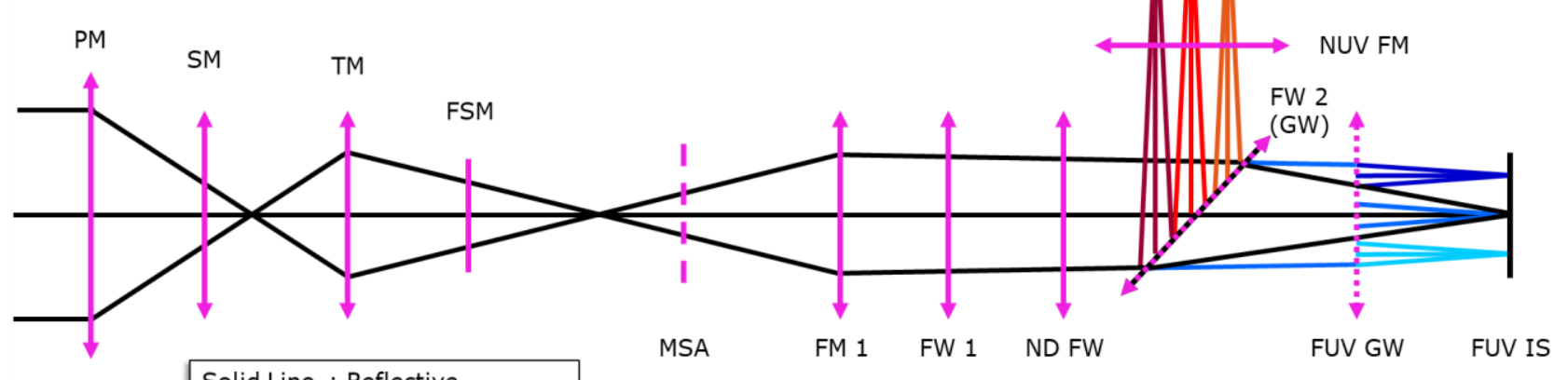

\begin{tabular}{|ll|}
\hline Solid Line & Reflective \\
Dashed & : Microshutter Array \\
Dotted & : Reflective Grating \\
Arrowed & : Powered Optic \\
Plane & : Flat Optic \\
\hline
\end{tabular}

Figure 11. Optical block diagram for LUMOS-B.

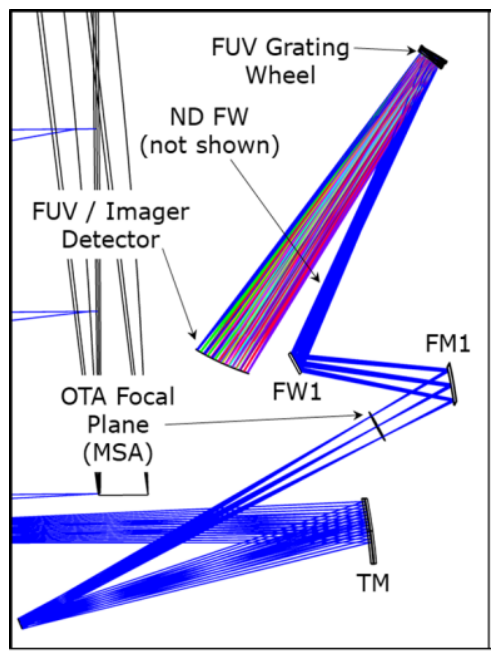

1. FUV multi-object spectrograph

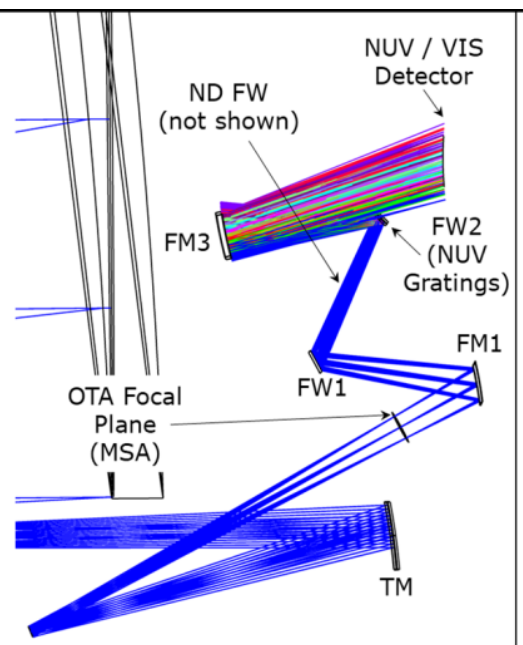

2. NUV/VIS multi-object spectrograph

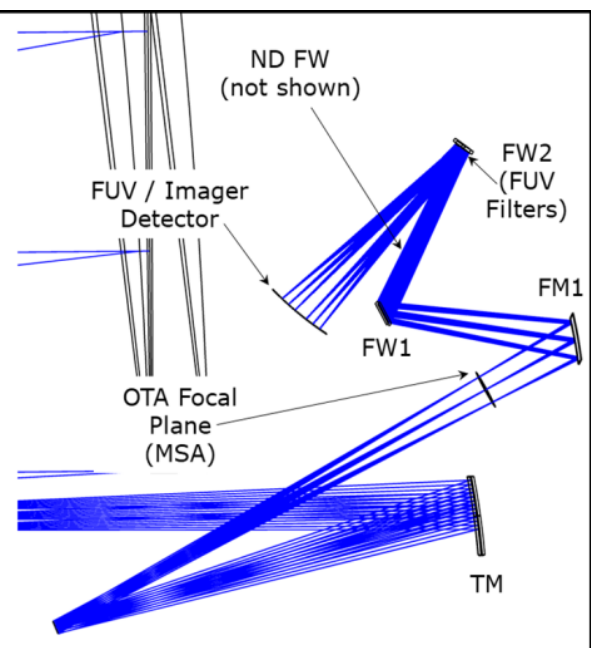

3. FUV imager

Figure 12. Layouts of the three individual channels for LUMOS-B. From left to right: far ultraviolet (FUV) multi-object spectrograph (MOS), near ultraviolet (NUV) MOS, and FUV imager.

\subsection{Summary of Performance}

Table 3 shows that, when averaged over the field of view, both of the current designs meet or exceed the $<50$ milli-arcsecond angular resolution constraint common to the FUV and NUV MOSs and the FUV imager.

Table 3 also shows that the majority of the spectral channel grating modes meet or exceed their resolving power specifications when averaged across the full field of view of the MSA. Those that do not are just under the requirement. All of the grating modes easily exceed the resolving power requirements when averaged over a smaller $1 \mathrm{x} 1$ ' field of view. To show an example of the results in more detail, Figure 13 displays the spectral and imaging performance of LUMOS-A's G150M mode as a function of field. As expected, both resolving power and angular resolution are best closer to the center of the field of view and degrade moving outwards to the edges. The equivalent analysis for LUMOS$\mathrm{B}$ is seen in Figure 14. For the FUV imaging channel, Figure 15 shows spot diagrams for both LUMOS-A and LUMOS- 
$\mathrm{B}$ at the center and corners of the field of view. The spot diagrams are calculated for a wavelength of $\lambda=100 \mathrm{~nm}$ (the shortest wavelength for the imaging channel). Future design iterations of LUMOS can be carried out to further improve performance in terms of both angular resolution and resolving power as a function of field.
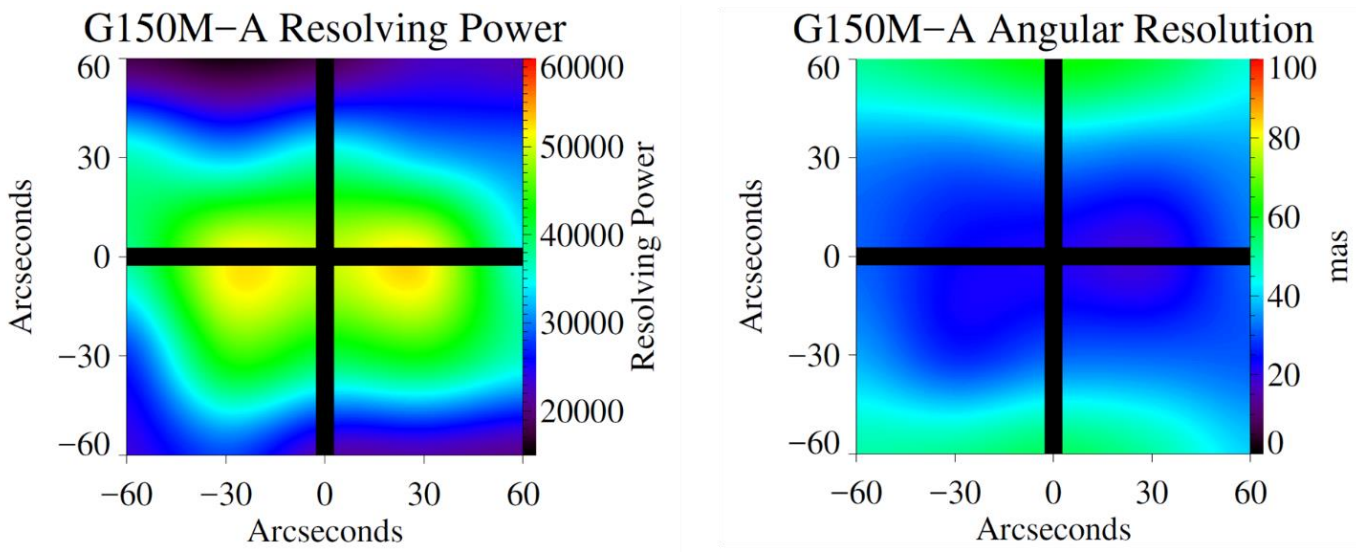

Figure 13. Spectral resolving power and angular resolution (in milli-arcseconds) maps across the FUV MOS focal plane (using the G150M mode as a representative example) for LUMOS-A. Gaps in the focal plane are created where the MSA arrays are tiled. The " $y$ " axis is the dispersion direction and the " $x$ " axis is the cross-dispersion direction in these plots.
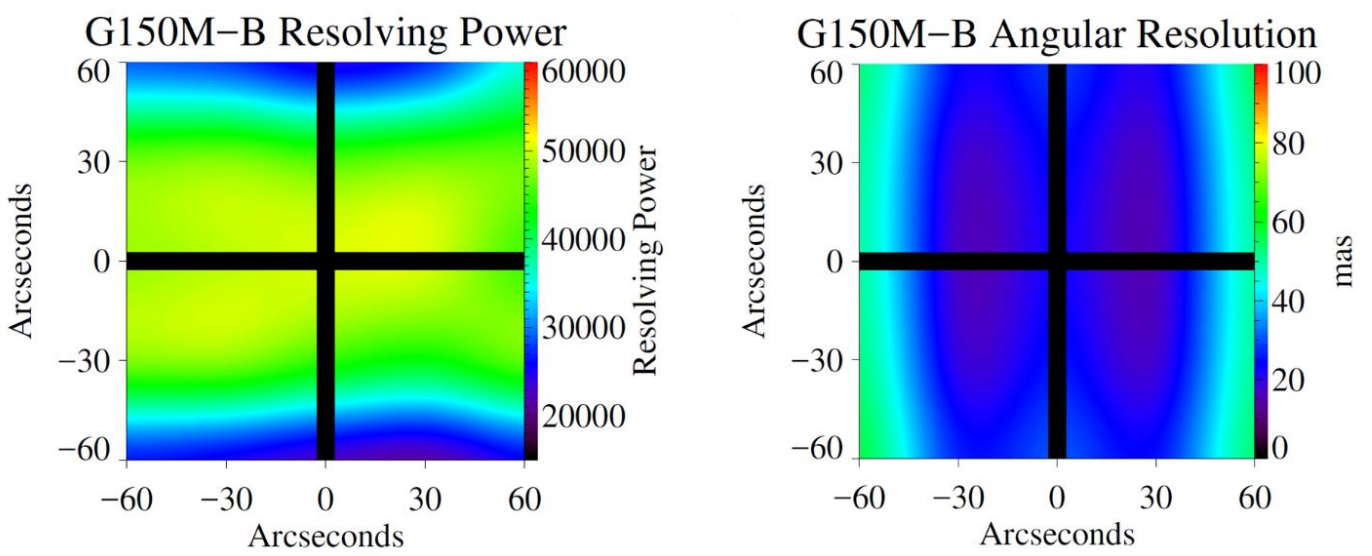

Figure 14. Spectral resolving power and angular resolution (in milli-arcseconds) maps across the FUV MOS focal plane (using the G150M mode as a representative example) for LUMOS-B. Gaps in the focal plane are created where the MSA arrays are tiled. The " $y$ " axis is the dispersion direction and the " $\mathrm{x}$ " axis is the cross-dispersion direction in these plots.
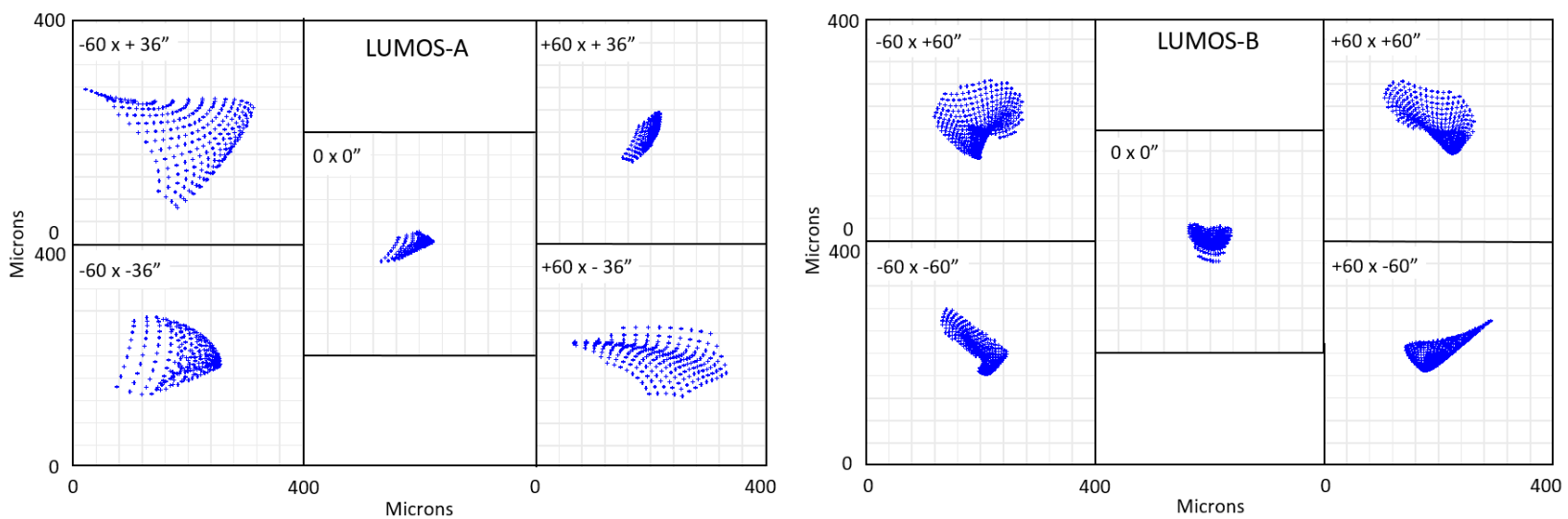

Figure 15. Spot diagrams for LUMOS-A (left) and LUMOS-B (right) across the 1.2 x 2 arcminute and 2 x 2 arcminute full fields of view, respectively. 


\section{EXTREME CORONAGRAPH FOR LIVING PLANETARY SYSTEMS (ECLIPS)}

\subsection{Overview}

LUVOIR's Extreme Coronagraph for Living Planetary Systems (ECLIPS) instrument is a high-contrast coronagraph that will be used to find and characterize a wide range of exoplanets. The instrument spans a wide waveband between 200 and 2000nm and is broken into three separate channels: ultraviolet (UV), visible (VIS), and near infrared (NIR). Each channel contains up to two modules. There are cameras within the UV and VIS channels, integral field spectrographs (IFS) within the VIS and NIR channels, and a single point-source spectrometer (SPSS) within the NIR channel. This section discusses the layout of the ECLIPS instrument.

\subsection{Requirements and Assumptions}

Coronagraphy with a large-aperture telescope is desirable for detecting exoplanets. Such telescopes increase the amount of photons collected, thereby increasing the signal-to-noise ratio (SNR) of the system, while also enabling a smaller inner working angle (IWA). The IWA scales with the parameter $\lambda / \mathrm{D}$ where $\mathrm{D}$ is the aperture diameter and $\lambda$ is the wavelength of light so that a larger diameter telescope is able to detect exoplanets closer to their host stars that a smaller diameter telescope may miss. This fact illustrates the importance of the large diameters of the LUVOIR-A and LUVOIR-B telescopes ( $15 \mathrm{~m}$ and $8 \mathrm{~m}$, respectively). The versions of the instrument that correspond to each telescope are referred to as ECLIPS-A and ECLIPS-B. The requirements for the coronagraph and each of its five modules are laid out in Table 4

Table 4. Optical design parameters of the ECLIPS instrument. Both versions of the instrument have the same specifications.

\begin{tabular}{|c|c|c|c|c|}
\hline \multirow{2}{*}{ Parameter } & \multicolumn{3}{|c|}{ Value } & \multirow{2}{*}{ Units } \\
\hline & UV & VIS & NIR & \\
\hline Raw Contrast & \multicolumn{3}{|c|}{$1 \times 10-10$} & - \\
\hline Total Bandpass & $200-525$ & $515-1030$ & $1000-2000$ & $\mathrm{~nm}$ \\
\hline Inner Working Angle & $4 N / D$ & $3.5 \mathrm{~N} / \mathrm{D}$ & $3.5 \mathrm{ND}$ & - \\
\hline Outer Working Angle & $40 \mathrm{~N} / \mathrm{D}$ & $64 N / D$ & $64 N / D$ & - \\
\hline RMSWavefront Error & 14 & 37 & 71 & $\mathrm{~nm}$ \\
\hline \multicolumn{5}{|c|}{ Spectrometer Lenslet Array Parameters } \\
\hline Sampling at Lenslet Array & - & Nyquist at $515 \mathrm{~nm}$ & Nyquist at $1000 \mathrm{~nm}$ & - \\
\hline Lenslet Diameter & - & 124 & 120 & $\mu \mathrm{m}$ \\
\hline Pinhole Diameter & - & 30 & 40 & $\mu \mathrm{m}$ \\
\hline Lenslet Array Size & - & $40 \times 40$ & $44 \times 44$ & $\mathrm{~mm}$ \\
\hline Lenslet Packing & - & Square & Hexagonal & - \\
\hline Total Lenslet Count & - & 72,300 & 128,000 & - \\
\hline $\mathrm{F} / \#$ at Lenslet Array & - & 481.5 & 248.0 & - \\
\hline \multicolumn{5}{|c|}{ Integral Field Spectrometer Detector Parameters } \\
\hline Sampling at Detector & - & Nyquist at $515 \mathrm{~nm}$ & Nyquist at $1000 \mathrm{~nm}$ & - \\
\hline Pixel Size & - & 12 & 10 & $\mu \mathrm{m}$ \\
\hline Magnification & - & $1: 1.47$ & 1:01 & - \\
\hline F/\# at Detector & - & 11.8 & 8.0 & - \\
\hline Total Spectral Length* & - & 34 & 20 & pixels \\
\hline Resovling Power & - & 140 & 70 & \\
\hline \multicolumn{5}{|c|}{ 'High-resolution Slit Spectrometer } \\
\hline Pixel Size & - & - & 10 & $\mu \mathrm{m}$ \\
\hline F/\# at Detector & - & - & 8.0 & - \\
\hline Resovling Power & - & - & 200.0 & - \\
\hline \multicolumn{5}{|c|}{ Imaging Camera Parameters } \\
\hline Sampling at Detector & Nyquist at $200 \mathrm{~nm}$ & Nyquist at $515 \mathrm{~nm}$ & - & - \\
\hline $\mathrm{F} / \#$ at Detector & 130.0 & 50.5 & - & - \\
\hline Effective Focal Length & 2,628 & 1,020 & - & $\mathrm{mm}$ \\
\hline Pixel Size & 13 & 13 & - & $\mu \mathrm{m}$ \\
\hline
\end{tabular}

*Includes isolation pixels 


\subsection{System Description}

Figure 16 shows a block diagram for the ECLIPS instrument, which is identical for both versions of the instrument excepting a minor difference in the order of the pupil relay optics that pick-off the beam from the OTA. In the current design of the ECLIPS instruments, three mirrors are used to pick-off the light from the OTA focal plane, collimate it, and re-image the telescope pupil onto the first deformable mirror (DM) in each channel. Once the light is collimated by these pre-optics, it is separated into each channel via a series of dichroic beamsplitters. The mirrors in each channel are coated according to their bandpass to provide the best reflectivity: the pre-optics and UV channel mirrors are coated in aluminum, the visible channel mirrors are coated in silver, and the NIR channel mirrors are coated in gold. To minimize polarization aberration effects within the instrument, all $90^{\circ}$ fold mirrors are used in crossed-pairs, such that one mirror compensates the polarization effects of the other. There is one exception to this in ECLIPS-B, where a single, uncompensated compound fold mirror was needed to orient the optical system in the available volume. However, we believe with additional design iterations this mirror can either be removed entirely, or compensated by an additional fold mirror to remove the induced polarization aberration.

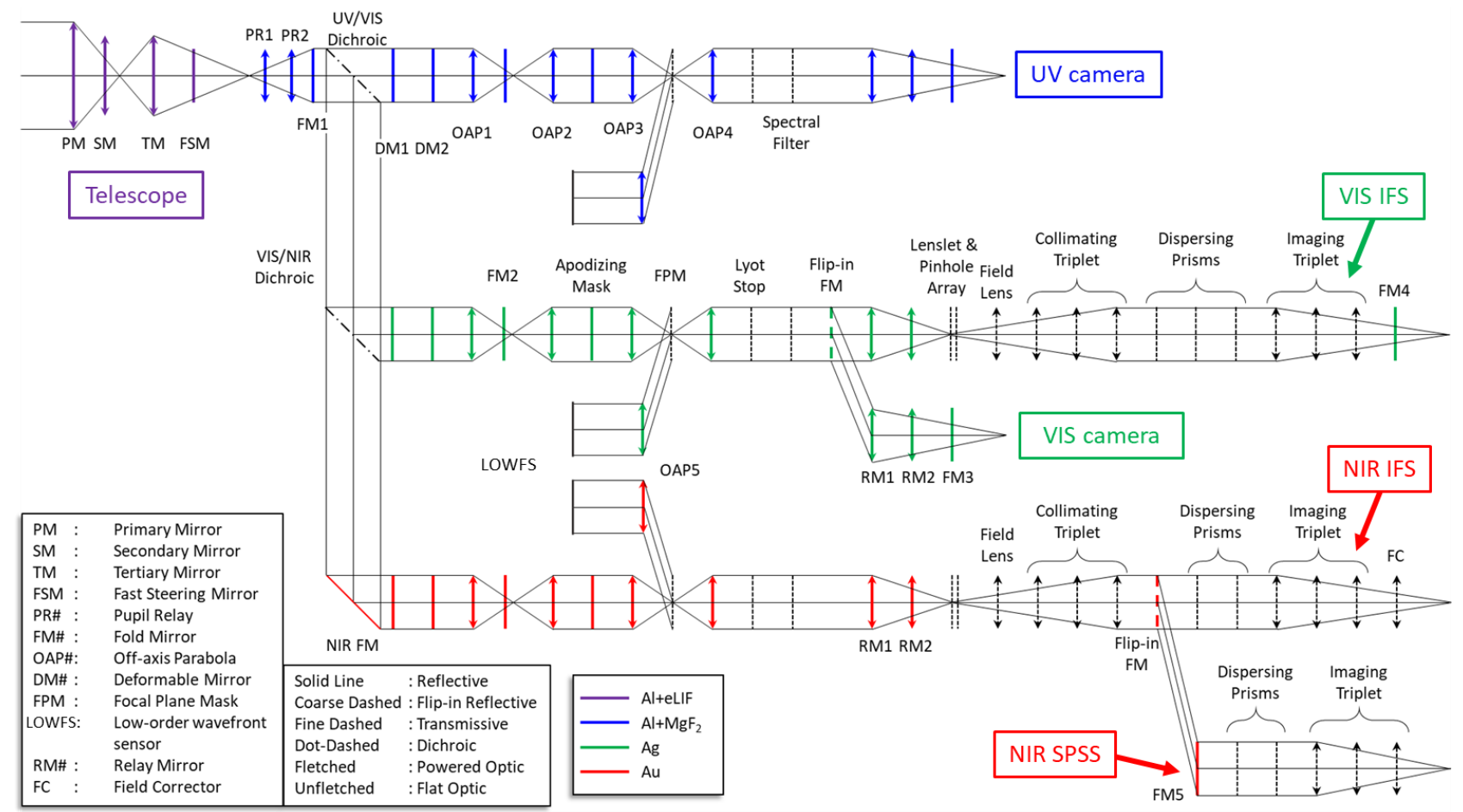

Figure 16. Optical block diagram for ECLIPS-A. The block diagram for ECLIPS-B is identical save for differences in the pre-optics between the telescope and instrument.

After the first DM, the beam propagates to a second DM that is not in a pupil plane. A pair of relay mirrors then reimage the pupil from the first DM onto the apodizing mask. A second pair of relay mirrors re-images the pupil again onto the Lyot stop, with the coronagraph focal plane mask is located at the intermediate focus. After passing through the Lyot stop, the optical bandpass is further reduced to $10-20 \%$ by spectral filters for science observations. Figure 17 shows the optical designs in Zemax for both ECLIPS-A and ECLIPS-B. Note the large distance between the instrument and its pre-optics for the B design. This was necessitated by the smaller volume afforded by LUVOIR-B as opposed to LUVOIR-A which required ECLIPS be placed as far from the other two instruments as possible to have enough room. Figure 17 also shows an example of a single coronagraph channel with no modules as described in the paragraph above. Each of the three spectral channels has its own set of optics consistent with that design (from dichroic to Lyot stop).

Depending on the channel, after passing through the spectral filters, the light can be sent to one of several back-end instruments. In the UV channel, the only back-end instrument is an imaging camera. In the visible channel, a flip-in mirror can be used to direct the light to either an imaging camera or an R=140 IFS. In the NIR channel, a flip-in mirror can be used to direct the light to either an R=70 IFS, or an R=200 SPSS. Each of the five modules meet their individual 
optical performance requirements. ${ }^{[5]}$ In addition to the primary science beam path, each channel also supports a low-order / out-of-band wavefront sensor (LOWFS/OBWFS).

Figure 18 shows an opto-mechanical model of ECLIPS-A. Two optical benches hold the three channels of the instrument. An interface bench at the base of the instrument holds the two optical benches and interfaces to the BSF bulkhead. The benches are aluminum honeycomb panels with composite face sheets. Additional structural support is provided by composite gussets and a central, cylindrical hub that braces the two benches near the instrument center-ofmass. The ECLIPS-B design is similar, save small modifications to accommodate packaging differences within the LUVOIR-B instrument volume.

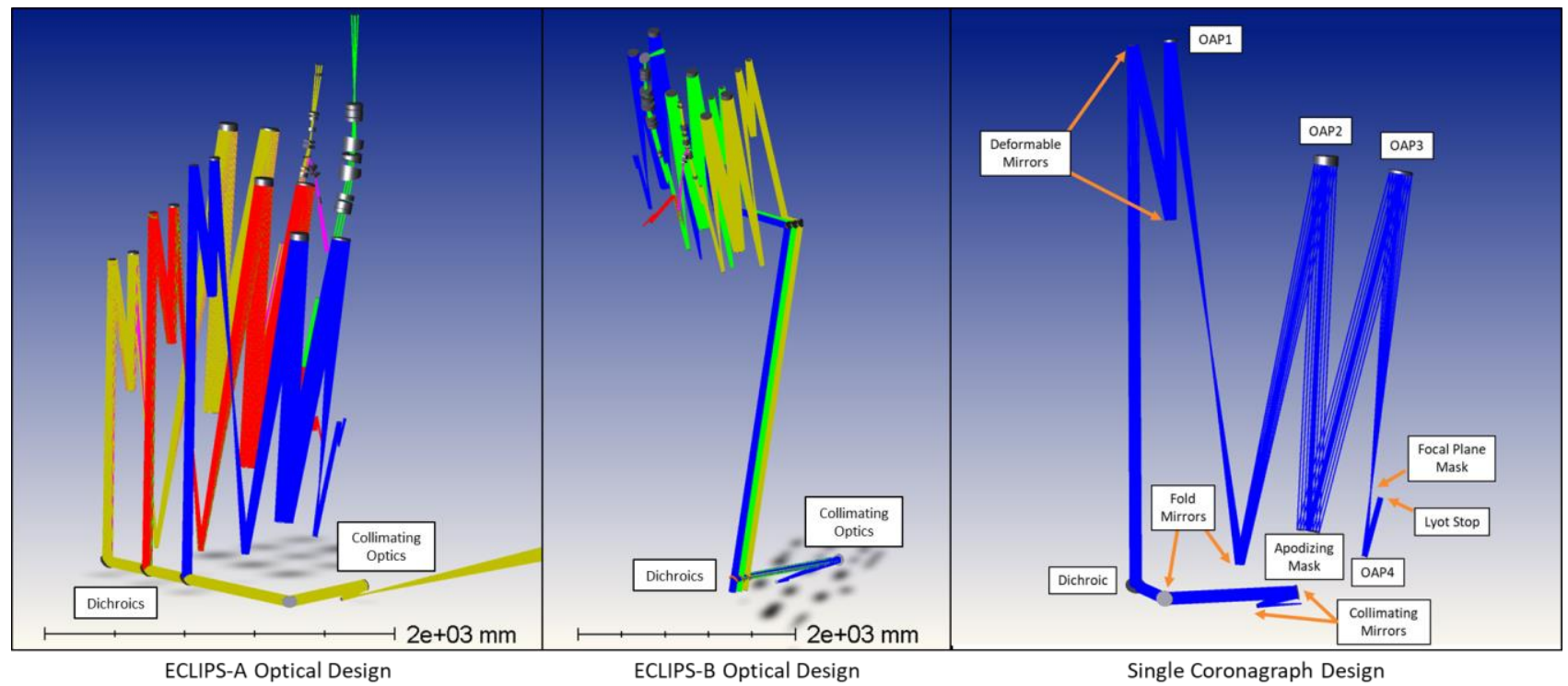

Figure 17. Optical designs for both ECLIPS-A and ECLIPS-B as well as layout for a single coronagraph channel with no modules.

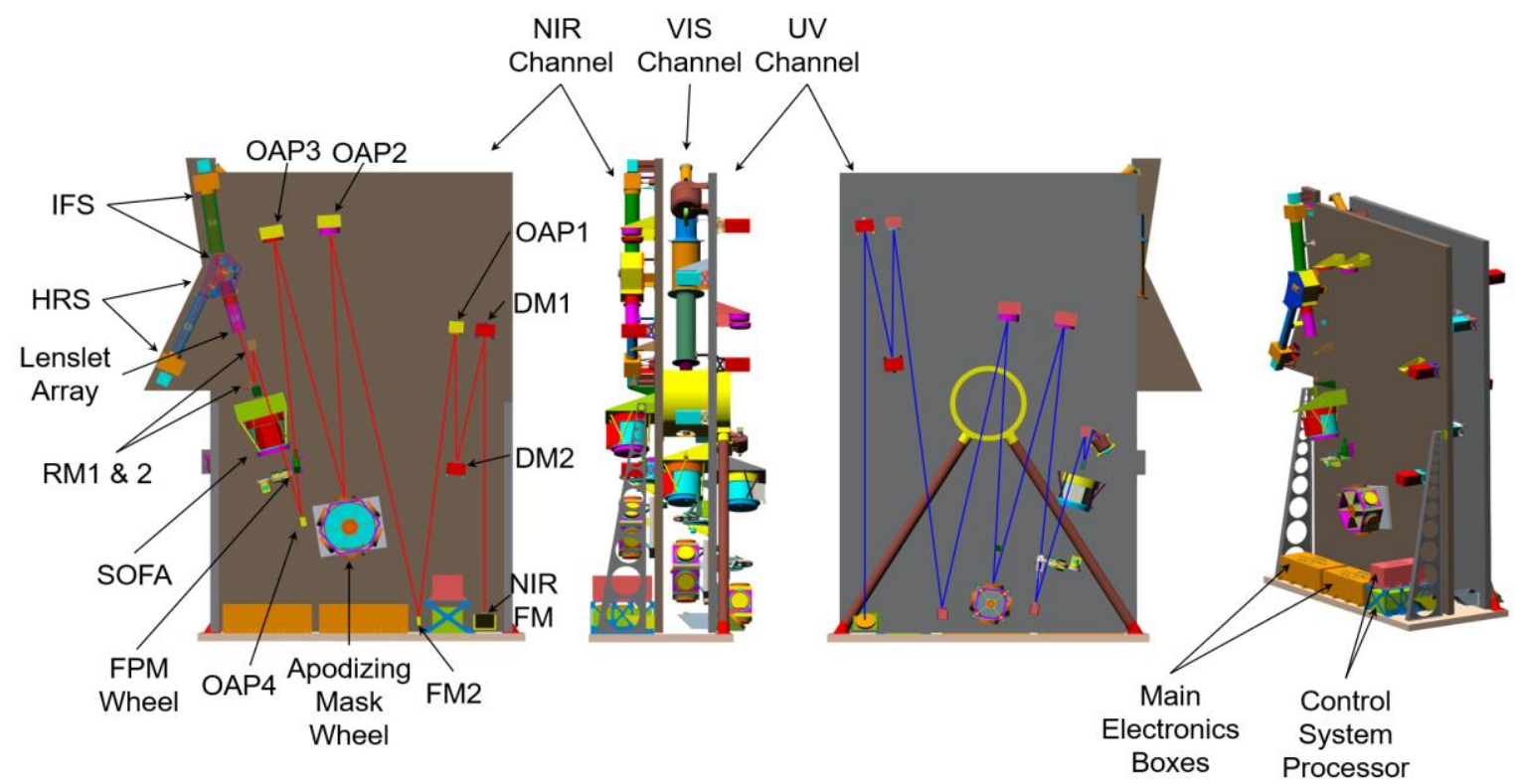

Figure 18. ECLIPS-A opto-mechanical design. The three channels are separated by two optical benches that are mounted to a third interface bench. Elements in the NIR channel (left) are identified using the same nomenclature as Figure 923 . The UV channel layout is similar, except after the Selectable Optical Filter Assembly (SOFA), the beam is relayed directly to an imaging camera. The Visible channel has a similar layout to the NIR channel, except there is no HRS, and the beam can instead be relayed to an imaging camera after the SOFA. The ECLIPS-B opto-mechanical design is very similar. 


\section{CONCLUSIONS}

In this paper, we presented an overview of the optomechanical designs for each of the three LUVOIR instruments: HDI, LUMOS, and ECLIPS. Optical and mechanical models exist for both the A and B variants of each instruments. All designs meet optical performance requirements while staying within allocations for mass and power. For more information on these topics, the reader is encouraged to read the LUVOIR Final Report, which details the results of this 3.5-year study, and is publicly available at https:/www.luvoirtelescope.org.

\section{REFERENCES}

[1] The LUVOIR Study Team, "The LUVOIR Final Report," available at https://www.luvoirtelescope.org/, 2019.

[2] Roberge, A. and Bolcar, M.R., "Telling the story of life in the cosmos: The LUVOIR telescope concepts," Proc. SPIE 11115, 1111523 (2019).

[3] Bolcar, M.R., "The Large UV/Optical/Infrared Surveyor (LUVOIR): Engineering Design \& Technology Overview," Proc. SPIE 11115, 1111524 (2019).

[4] France, K., Fleming, B, West, G. et al., "The LUVOIR Ultraviolet Multi-Object Spectrograph (LUMOS): instrument definition and design," Proc. SPIE 10397, UV, X-Ray, and Gamma-Ray Space Instrumentation for Astronomy XX, 1039713 (2017);

[5] Gong, Q., Bolcar, M.R., Corsetti J.A et al, "Optical design of the Extreme Coronagraph for Living Planetary Systems instrument for the LUVOIR mission study," J. Astron. Telesc. Instrum. Syst. 5(2) 025002 (9 May 2019) 